\title{
BMJ Open Acupotomy versus acupuncture for cervical spondylotic radiculopathy: protocol of a systematic review and meta-analysis
}

\author{
Bin Chen, ${ }^{\oplus}$ Cai Zhang, ${ }^{2}$ Ren-Pan Zhang, ${ }^{1,2}$ An-Yang Lin, ${ }^{1,2}$ Zhong-Biao Xiu, ${ }^{1}$ \\ Jing Liu, ${ }^{1}$ Hong-Jia Zhao ${ }^{1}$
}

To cite: Chen B, Zhang $C$, Zhang R-P, et al. Acupotomy versus acupuncture for cervical spondylotic radiculopathy: protocol of a systematic review and meta-analysis. BMJ Open 2019;9:e029052. doi:10.1136/ bmjopen-2019-029052

- Prepublication history for this paper is available online. To view these files please visit the journal online (http://dx.doi org/10.1136/bmjopen-2019029052).

Received 10 January 2019

Revised 21 June 2019 Accepted 1 July 2019

A) Check for updates

(C) Author(s) (or their employer(s)) 2019. Re-use permitted under CC BY-NC. No commercial re-use. See rights and permissions. Published by BMJ.

${ }^{1}$ Department of Rehabilitation, The Affiliated People's Hospital, Fujian University of Traditional Chinese Medicine, Fuzhou, China

${ }^{2}$ Graduate School, Fujian University of Traditional Chinese Medicine, Fuzhou, China

Correspondence to Professor Hong-Jia Zhao; hongjiafz@163.com

\section{ABSTRACT}

Introduction Cervical spondylotic radiculopathy (CSR) is the most common pattern of cervical spondylosis, which is a serious and common degenerative disease. Both acupotomy and acupuncture have been widely used clinically to treat CSR in China with satisfied efficacy. However, there is no systematic review comparing the effectiveness of these two therapies. The aim of this study is to compare the therapeutic efficacy and safety between acupotomy and acupuncture for patients with CSR to provide evidence for clinical practice.

Methods and analysis The following electronic databases will be searched: Web of Science, PubMed, Embase, Cochrane Library, China National Knowledge Infrastructure, China Biology Medicine disc, Wanfang Database and Chinese Scientific Journal Database (VIP). The randomised controlled trials of acupotomy versus acupuncture with/without additional treatment for CSR will be searched in the databases from their inception to December 2018 by two researchers independently. Visual analogue scale, symptom score and neck disability index will be assessed as the primary outcomes. The total effective rate, curative rate, adverse events and amount of rescue medication used will be assessed as the secondary outcomes. The Review Manager 5.3 will be used for metaanalysis and the evidence level will be assessed by using the method for Grading of Recommendations Assessment, Development and Evaluation. Continuous outcomes will be presented as the weighted mean difference or standardised mean difference with $95 \% \mathrm{Cl}$, whereas dichotomous data will be expressed as relative risk with $95 \% \mathrm{Cl}$. If the included studies have existing heterogeneity $(p<0.05)$, then a random-effects model will be used. Otherwise, we will calculate using a fixed-effects model. Ethics and dissemination Ethical approval is not required because no primary data are collected. This review will be published in a peer-reviewed journal and will be presented at an international academic conference for dissemination.

PROSPERO registration number CRD42019117348.

\section{INTRODUCTION}

Cervical spondylotic radiculopathy (CSR) is defined as neck pain in a radicular pattern in one or both upper extremities related to
Strengths and limitations of this study

- This systematic review will comprehensively compare the therapeutic efficacy and safety between acupotomy and acupuncture for cervical spondylotic radiculopathy.

- The study screening, data extraction and quality assessment will be performed by two independent reviewers.

- Different types of acupuncture and some of the reviewed trials with small sample sizes may cause considerable heterogeneity in this review. Highquality trials might be deficient to generate convincing conclusions.

compression and/or irritation of one or more cervical nerve roots. The most common level of nerve root compression is $\mathrm{C} 7$, followed by C6. CSR, accounting for about $60 \%-70 \%$ of all cervical spondylosis, is the common and frequently occurring disease in both middleaged and elderly populations. ${ }^{1}$ It has been reported that up to $80 \%$ of cervical spondylosis patients always complained of neck pain, which will become more and more serious over time. Due to social technology advances, lifestyle changes and the increase in staff members, the incidence of CSR tends to increase and the onset age of the patient gets younger year by year, which has seriously affected the patients' physical health and quality of life.

Management of CSR can be surgical or conservative. Conservative management is the initial treatment of choice for most patients, because surgery may be associated with adverse events and recurrence. Currently, many therapeutic interventions have been applied for the treatment of CSR, including non-steroidal anti-inflammatory drugs (NSAIDs), ${ }^{2}$ epidural steroid injections, ${ }^{3}$ acupuncture, ${ }^{4}$ physical therapy ${ }^{5}$ and 
exercises. ${ }^{67}$ The main purpose of these treatments is to relieve pain, improve function and enhance the quality of life. In clinical practice, acupotomy has also been widely used to treat cervical spondylosis, lumbar disc herniation, knee osteoarthritis and other diseases in China with satisfied efficacy, because of its effectiveness and low risk of complications. $^{8-12}$

Acupotomy, also named needle knife, originates from the 'nine classical of needles' in Huang Di Nei Jing (Huangdi's Internal Classic) and was developed in China in 1976 by Zhu Hanzhang. ${ }^{13}$ It is a new-style bladed needle that composed of a thick flat-head and a cylindrical body, which is suitable for alleviating the adhesion of a lesion. Acupotomy therapy is considered as a minimally invasive surgery of traditional Chinese medicine, combining Chinese acupuncture therapy and modern surgical principles. ${ }^{14}$ In the treatment of CSR, the role of acupotomy is to remove attached tissues, recover the dynamic function of soft tissues, relieve nerve pressure and promote Qi-blood circulation to ameliorate pain and numbness symptoms. ${ }^{10} 15$ Acupotomy has many benefits because it converts open surgery to closed surgery, thus reducing risk, time and cost. This method only produces a small scar that will fade with time. ${ }^{11}$

Acupotomy has the characteristics of both acupuncture and microinvasive operation. Both acupotomy and acupuncture have been widely used clinically to treat CSR in China with satisfied efficacy. However, there is no systematic review comparing the effectiveness of these two therapies in patients with CSR. It is worthy to critically review the evidence of the comparison of these two therapies to inform clinical practice. Herein, the aim of this study is to compare the therapeutic efficacy and safety between acupotomy and acupuncture on CSR to provide evidence for clinical practice.

\section{METHODS}

\section{Study registration}

This systematic review and meta-analysis protocol was registered in PROSPERO (CRD42019117348) at https:// www.crd.york.ac.uk/PROSPERO/\#myprospero.

\section{Inclusion criteria for study selection}

Type of study

We will estimate the research literature according to the criteria of the review objectives and participants, interventions, comparisons, outcomes (PICO). Randomised controlled trials (RCTs), comparing acupotomy against any form of acupuncture with/without additional treatment, will be included in this systematic review. We will include such studies if the expression 'randomisation' is mentioned. However, we will grade these studies as high in the 'risk of bias assessment' if the detailed description of the randomisation process is not provided. Furthermore, if an incorrect randomisation method such as coin toss was used, the study will not be included. Moreover, blinding will not be considered because of the characteristics of acupuncture and acupotomy treatment. Additionally, the language of the publications will be limited to Chinese and English.

\section{Types of participants}

Patients of any gender or age or race or nationality with CSR received acupotomy or acupuncture therapy with/ without additional treatment.

1. In line with the diagnostic criteria of CSR.

2. Participants who have not undergone an invasive intervention.

3. No restriction on age.

The exclusion criteria were shown as follows: (1) replicated studies; (2) no definite diagnostic criteria of CSR; (3) wrong interventions: these studies were excluded which used open surgery or acupotomy was manipulated in both groups; (4) reviews or theory studies; (5) animal experiments.

\section{Types of interventions}

\section{Experimental interventions}

The treatment group will be treated with acupotomy (with/without additional treatment). No restrictions are imposed on times of treatment, frequency of treatment, and length of the treatment period.

\section{Comparator interventions}

The treatment with several types of acupuncture (with/ without same additional treatment) will be included in this review as acupuncture, manual acupuncture, auricular acupuncture, scalp acupuncture, fire needling, warm needling, electroacupuncture and so on.

The procedure of acupuncture and acupotomy should be reported in full compliance with the standardised reporting methods such as the Standard of the Basic Manipulations of Acupotomy (ZJ/T D001-2014) and Standards for Reporting Interventions in Controlled Trials of Acupuncture (STRICTA).

\section{Types of outcome measures}

Changes in visual analogue scale (VAS), symptom score and neck disability index (NDI) will be evaluated as primary outcomes. The total effective rate and the curative rate will be evaluated as secondary outcomes. The secondary outcome measures are as follows:

1. Total effective rate and curative rate

The total effective rate and curative rate are non-validated outcome measures that are processed secondarily according to certain evaluation criteria such as clinical symptom improvement or the improvement rates of other quantified outcomes. In the assessment of the total effective rate, participants are generally classified as 'cured', 'markedly improved', 'improved' or 'non-responder' after treatment. The total effective rate is calculated consistently using the following formula:

$$
\text { Total effective rate }=N_{1}+N_{2}+N_{3} / N
$$




$$
\text { Curative rate }=N_{1} / N
$$

where N1, N2, N3 and N are the number of patients who are cured, markedly improved, improved and who comprise the sample size, respectively.

2. The incidence of adverse events.

3. The amount of rescue medication required.

\section{Data sources}

The main sources of information to be obtained in this study include electronic resource databases, trial registries, retroactive references and different types of grey literature.

\section{Electronics searches}

The following electronic databases will be searched: Web of Science, PubMed, Embase, Cochrane Library, China National Knowledge Infrastructure (CNKI), China Biology Medicine disc (CBM), Wanfang Database and Chinese Scientific Journal Database (VIP). In addition, 'grey literature' such as conference proceedings and theses will be allowed. Reference lists of the relevant literature and systematic reviews, as well as the tables of contents related to acupotomy versus acupuncture on CSR, will also be searched. RCTs of acupotomy versus acupuncture with/without additional treatment for patients with CSR will be searched in the databases from their inception to December 2018 by two researchers independently.

\section{Searching for other resources}

Ambiguous literature will be investigated manually to avoid missing eligible trials. Reference lists of identified publications will also be manually searched. In addition, the following journals published in Chinese will be searched as a supplement: Chinese Acupuncture and Moxibustion (1981-December 2018), Acupuncture Research (1976-December 2018), World Journal of Acupuncture-moxibustion (1991-December 2018), Journal of Clinical Acupuncture and Moxibustion (1985-December 2018), Shanghai Journal of Acupuncture and Moxibustion (1982-December 2018) and Journal of Traditional Chinese Medicine (1960December 2018).

\section{Search strategy}

The strategy will be created according to the Cochrane handbook guidelines. The established search strategy for PubMed was displayed, as follows:

Mesh term \#1: ((acupotomy) OR (acupotome) OR (needle knife) OR (needle scalpel) OR (acupotomlogy) OR (miniscalpel acupuncture) OR (miniscalpel needle) OR (stiletto needle) OR (sword like needle) OR (Xiaozhendao)): ti, ab, kw.

Mesh term \#2: ((acupuncture) OR (manual acupuncture) OR (auricular acupuncture) OR (scalp acupuncture) OR (fire needling) OR (warm needling) OR (electro-acupuncture)): ti, ab, kw.

Mesh term \#3: ((cervical radiculopathy) OR (cervical spondylotic radiculopathy) OR (cervical spondylopathy)
OR (cervical spondylosis) OR (neck pain) OR (neck syndrome): ti, ab, kw.

Mesh term \#4: ((clinical trials) OR (random control trials)).

\section{\#1 AND \#2 AND \#3 AND \#4}

The equivalent search words will be used in Chinese databases.

\section{Data collection and analysis \\ Selection of studies}

The researchers will import the retrieved literature into an EndNote library and eliminate duplicate data. Two review authors (Renpan Zhang and Anyang Lin) selected studies for eligibility and checked against the inclusion criteria independently. Any disagreement will be resolved by consensus or consultation with a third independent researcher (Hongjia Zhao). The selection process is illustrated in a Preferred Reporting Items for Systematic review and Meta-Analysis flow diagram (figure 1).

\section{Data extraction and management}

Two review authors (Jing Liu and Zhongbiao Xiu) will independently use a standardised form for extracting data of the included articles. The following data were extracted: general information (eg, title, authors, year and published country), details of study (eg, design, inclusion and exclusion criteria, blinding, randomisation and sample size), participant characteristics (eg, age and number of subjects), description of interventions, types of outcomes assessed, adverse events and other detailed information. If necessary, we will contact the corresponding authors of trials as much as possible for further information.

Assessment of risk of bias and reporting of study quality

The Cochrane risk of bias tool will be applied to evaluate the quality and risk of bias in the ultimately included studies by two authors (Renpan Zhang and Anyang Lin) independently. ${ }^{16}{ }^{17}$ Risk of bias assessment categories will include the following: (1) random sequence generation; (2) allocation concealment; (3) blinding of participants; (4) blinding of outcome assessors; (5) completeness of outcome data; (6) selective outcome reporting and (7) other biases. The assessments for each item will be graded as low risk, unclear risk and high risk to evaluate several risks of bias that can occur in RCTs. In the case of other sources of bias, it was evaluated as 'low' if the characteristics of participants in each group were reported to be statistically homogeneous at baseline, but was otherwise rated 'high'. The results were presented as a risk of bias graph and risk of bias summary using the Cochrane Collaboration's software programme Review Manager (RevMan) version 5.3 for Windows (Copenhagen, The Nordic Cochrane Centre, the Cochrane Collaboration, 2012). If there is any disagreement take place, then the arbiter (Hongjia Zhao) will do the final judge. 


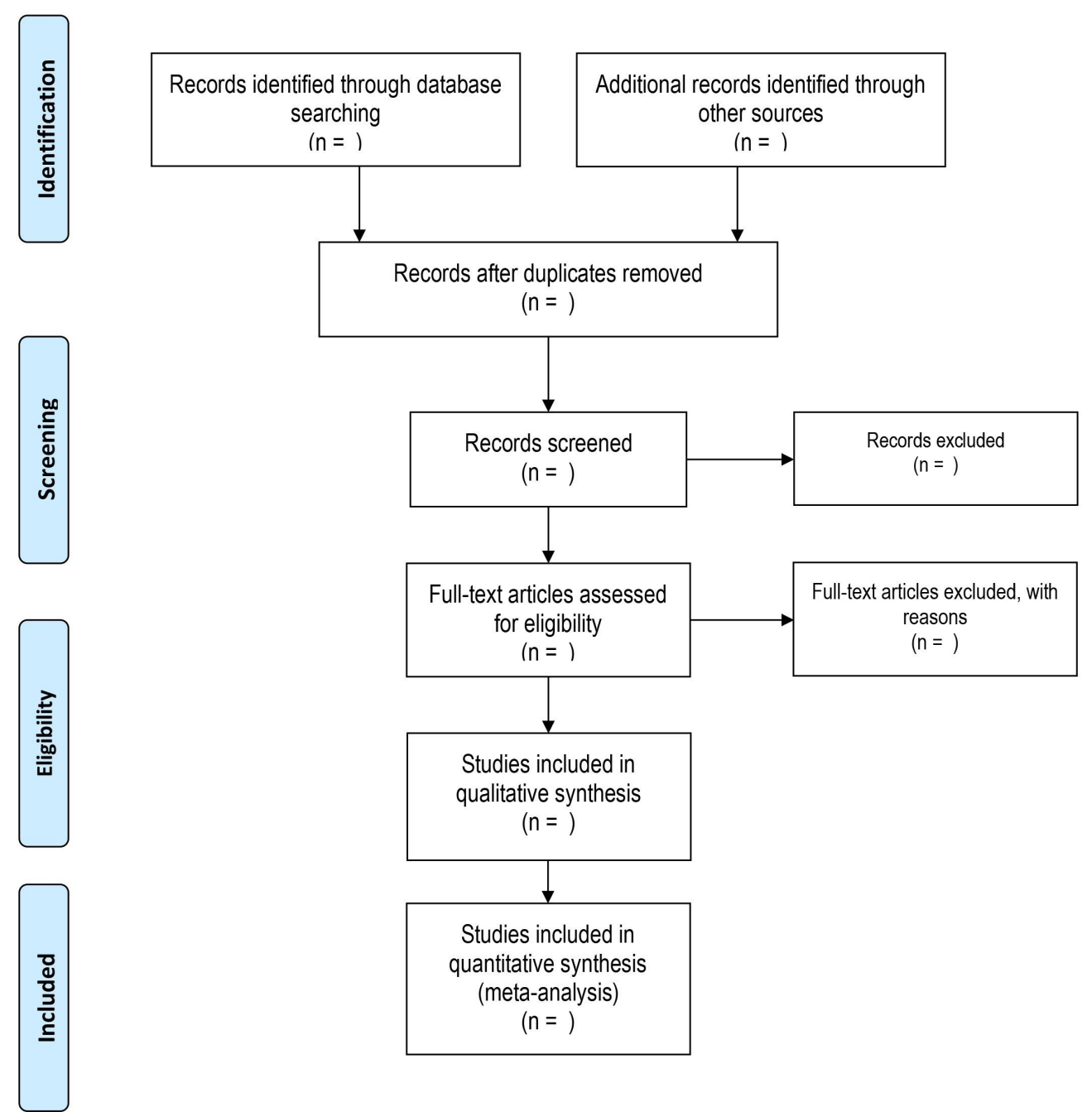

Figure 1 PRISMA flow diagram of the study process. PRISMA, Preferred Reporting Items for Systematic review and MetaAnalysis.

\section{Measures of treatment effect}

Continuous outcomes will be presented as the mean difference (MD) for analysis, while dichotomous data will be expressed as relative risk (RR), both of them will be with $95 \%$ CI. ${ }^{18}$ When the same outcome is measured in different ways, the standardised mean difference (SMD) with $95 \%$ CI will be selected to express the size of the intervention effect.

\section{Dealing with missing data}

We will attempt to contact authors of included studies for missing or incomplete data by email. However, if the missing data cannot be obtained, then the study will be excluded from the analysis.

\section{Assessment of heterogeneity}

Statistical heterogeneity will be detected by the $I^{2}$ statistic and $\chi^{2}$ test. $\mathrm{P}<0.1$ of the $\chi^{2}$ test or $I^{2}>50 \%$ indicates the possibility of statistical heterogeneity among the studies. If the included studies have existing heterogeneity, a random-effects model will be used. Otherwise, we will use a fixed-effect model for calculation.
Assessment of reporting bias

If $>10$ studies are included, then visual asymmetry on the funnel plots will be used to assess the potential reporting biases. In addition, we will test asymmetry using the Harbord modified test for dichotomous outcomes and Egger test for continuous outcomes.

\section{Data synthesis}

Review Manager 5.3 will be employed for meta-analysis. When statistical heterogeneity is low among the results, the fixed-effects model will be used for the meta-analysis. However, if there is considerable heterogeneity, then the random-effects model will be used to analyse the pooled effect estimates.

\section{Subgroup analysis}

If there is significant heterogeneity in the included trials, then we will conduct a subgroup analysis based on the acupotomy or acupuncture interventions with/without additional treatment, types of acupuncture (acupuncture, manual acupuncture, electroacupuncture and so on) and different outcomes. 


\section{Sensitivity analysis}

A sensitivity analysis will be conducted to identify whether the review conclusions are robust according to the following criteria: missing data, sample size, heterogeneity qualities and statistical model.

\section{Grading the quality of evidence}

The evidence level will be assessed by using the method for Grading of Recommendations Assessment, Development and Evaluation (GRADE) and classified into four possible ratings: very low, low, moderate or high.

\section{Patient and public involvement}

Patients and or the public were not involved because no primary data are collected.

\section{Ethics and dissemination}

Ethical approval will not be needed because no primary data are collected. Our results will provide clear evidence to determine whether acupotomy therapy is an effective and safe intervention for patients with CSR, and thus will be beneficial to patients, practitioners and policy-makers. This review will be published in a peer-reviewed journal and will be presented at an international academic conference for dissemination.

\section{DISCUSSION}

The main symptoms of CSR include neck and shoulder pain, radicular pain or numbness in the upper extremities, weakening of grip strength and sensory disturbances. Acupotomy, containing the characteristics of both acupuncture and microinvasive operation, has been widely used clinically to treat CSR by peeling synechia, removing attached tissue and relieving nerve pressure. Currently, there is limited evidence to determine whether acupotomy and acupuncture have a similar effect on relieving pain and improving other symptoms of CSR. Therefore, the comparisons of therapeutic efficacy and safety will be made between acupotomy and acupuncture with/without the same additional treatment is given to both groups. This systematic review and meta-analysis will provide high-quality evidence-based medicine to determine whether acupotomy therapy is an effective and safe intervention for patients with CSR.

Contributors $\mathrm{BC}$ and $\mathrm{H}-\mathrm{JZ}$ : designed the systematic review. $\mathrm{BC}$ and $\mathrm{CZ}$ : drafted the article. R-PZ, A-YL, JL and Z-BX: independently screen the potential studies, extract the data, assess the risk of bias and finish the data synthesis. H-JZ: arbitrate any disagreement and ensure that no errors occur during the review. All review authors critically reviewed, revised, and approved the subsequent and final version of the protocol.

Funding This study was funded by the National Natural Science Foundation of China (Grant N0.81804175 and 81873315) and Fujian Provincial Department of Education Applied Discipline-Clinical Medicine 'Double First Class' Construction Project.
Competing interests None declared.

\section{Patient consent for publication Not required.}

Provenance and peer review Not commissioned; externally peer reviewed.

Open access This is an open access article distributed in accordance with the Creative Commons Attribution Non Commercial (CC BY-NC 4.0) license, which permits others to distribute, remix, adapt, build upon this work non-commercially, and license their derivative works on different terms, provided the original work is properly cited, appropriate credit is given, any changes made indicated, and the use is non-commercial. See: http://creativecommons.org/licenses/by-nc/4.0/.

\section{REFERENCES}

1. Yang F, Li WX, Liu Z, et al. Balance chiropractic therapy for cervical spondylotic radiculopathy: study protocol for a randomized controlled trial. Trials 2016;17:513.

2. Kartha S, Weisshaar CL, Philips BH, et al. Pre-treatment with Meloxicam Prevents the Spinal Inflammation and Oxidative Stress in DRG Neurons that Accompany Painful Cervical Radiculopathy. Neuroscience 2018;388:393-404.

3. Kim EJ, Chotai S, Schneider BJ, et al. Effect of depression on patient-reported outcomes following cervical epidural steroid injection for degenerative spine disease. Pain Med 2018;19:2371-6.

4. Wei X, Wang S, Li J, et al. Complementary and alternative medicine for the management of cervical radiculopathy: an overview of systematic reviews. Evid Based Complement Alternat Med 2015;2015:1-10.

5. Zhu L, Wei X, Wang S. Does cervical spine manipulation reduce pain in people with degenerative cervical radiculopathy? A systematic review of the evidence, and a meta-analysis. Clin Rehabil 2016;30:145-55.

6. Cheng CH, Tsai LC, Chung HC, et al. Exercise training for nonoperative and post-operative patient with cervical radiculopathy: a literature review. J Phys Ther Sci 2015;27:3011-8.

7. Buyukturan B, Guclu-Gunduz A, Buyukturan O, et al. Cervical stability training with and without core stability training for patients with cervical disc herniation: A randomized, single-blind study. Eur $J$ Pain 2017;21:1678-87.

8. Liu F, Zhou F, Zhao M, et al. Acupotomy therapy for chronic nonspecific neck pain: a systematic review and meta-analysis. Evid Based Complement Alternat Med 2017;2017:1-12.

9. Li S, Shen T, Liang Y, et al. Effects of miniscalpel-needle release on chronic neck pain: a retrospective analysis with 12-month follow-up. PLoS One 2015; 10:e0137033.

10. Kim SY, Kim E, Kwon O, et al. Effectiveness and safety of acupotomy for lumbar disc herniation: a randomized, assessor-blinded, controlled pilot study. Evid Based Complement Alternat Med 2018;2018:1-7.

11. Kim HJ, Jeon JH, Kim YI. Clinical effect of acupotomy combined with korean medicine: a case series of a herniated intervertebral disc. $J$ Acupunct Meridian Stud 2016;9:31-41.

12. Ding $Y$, Wang $Y$, Shi $X$, et al. Effect of ultrasound-guided acupotomy vs electro-acupuncture onknee osteoarthritis: a randomized controlled study. J Tradit Chin Med 2016;36:450-5.

13. Jeong JK, Kim YI, Kim E, et al. Effectiveness and safety of acupotomy for treating back and/or leg pain in patients with lumbar disc herniation: A study protocol for a multicenter, randomized, controlled, clinical trial. Medicine 2018;97:e11951.

14. Guo C, Liu N, Li X, et al. Effect of acupotomy on nitric oxide synthase and beta-endorphin in third lumbar vertebrae transverse process syndrome model rats. J Tradit Chin Med 2014;34:194-8.

15. Jiang $\mathrm{C}, \mathrm{Li} \mathrm{Y}$, Guo M, et al. Acupotomy therapy for lumbar disc herniation: protocol for a systematic review and meta-analysis. Medicine 2018;97:e12624.

16. The Cochrane Collaboration. Chapter 8: assessing risk of bias in included studies. In: Higgins J, Green S, eds. Cochrane Handbook for Systematic Reviews of Interventions Version 5.1.0, 2011.

17. Kwon CY, Lee B, Chang GT, et al. Efficacy of acupotomy for cerebral palsy: a systematic review and meta-analysis. Medicine 2019;98:e14187.

18. Ye Y, Xiao LY, Liu YH, et al. Acupuncture for patients with vascular dementia: a systematic review protocol. BMJ Open 2017;7:e019066. 